\title{
The clinical and prognostic significance of HER2 and Ki67 expression in 2062 Chinese patients with resectable gastric cancer: a retrospective study
}



Research

Keywords: Human epidermal growth receptor 2, Ki67, Gastric cancer, Chinese population, Prognostic value

Posted Date: May 20th, 2020

DOI: https://doi.org/10.21203/rs.3.rs-29263/v1

License: (c) (i) This work is licensed under a Creative Commons Attribution 4.0 International License. Read Full License 


\section{Abstract \\ Background}

The purpose of this study was to assess the prognostic value of the expression of human epidermal growth factor receptor 2 (HER2), Ki67, and their combination levels in the prognosis of Chinese patients with resectable gastric cancer (GC).

\section{Methods}

A total of 2062 Chinese GC patients were recruited with HER2 and Ki67 expression being evaluated using immunohistochemistry. Patients were divided into four groups according to HER2 and Ki67 expression. The distributions between HER2 and Ki67 expression levels and clinicopathological characteristics were compared using the Chi-square test. The relationship between HER2 and Ki67 expression level and overall survival were evaluated with the univariate and multivariate Cox regression analysis.

\section{Results}

There was no statistical differences between the overall survival (OS) rate and the expression level of HER2 $(P=0.748)$ or Ki67 $(P=0.063)$, but there were significant relationships between the OS rates and the combining expression levels of HER2/Ki67 $(P<0.05)$. Further, Ki67, sex, T stage, $\mathrm{N}$ stage, TNM stage, and adjuvant chemotherapy were significant and independent risk factors for GC survival $(P<0.05)$.

\section{Conclusions}

Our study illustrated that Ki67, but not HER2 acted as an independent prognostic factor in Chinese resectable GC patients. The evaluation of the combining expression levels of HER2/Ki67 may be more useful to assess patient prognosis with resectable GC.

\section{Introduction}

Gastric cancer (GC) was the fifth most common malignancy in 2018, with 1,033,701 new cases (5.7\% of all cancers), and the third leading cause of cancer death worldwide, with 782,685 deaths (8.2\% of all cancer deaths). ${ }^{1}$ China accounts for nearly one-half of the world's GC cases with over 400,000 new cases and an estimated 290,000 deaths in $2014 .^{2}$ The 5-year survival rate of Chinese GC was 31.3\%, much lower than North Korea (58\%) and Japan (54\%), and even lower than Mauritius (41\%). ${ }^{3}$ The fundamental treatment of GC involves the complete resection of localized tumors, but recurrence and metastatic spread occur frequently, nevertheless. In recent years, although the development of new agents and combination chemotherapies has gradually improved, the overall survival (OS) of advanced GC patients and the effective treatment therapy of advanced GC, especially potential therapeutic targets, is still a great challenge.

Among targeted therapeutic drugs, trastuzumab, a humanized monoclonal antibody, was firstly approved by the US Food and Drug Administration (USFDA) for human epidermal growth factor receptor 2 (HER2)-targeted therapy to treat HER2-positive breast cancer in $1998 .{ }^{4}$ Over the last decade, trastuzumab application has significantly improved the outcomes of HER2-positive breast tumors, which reinforces the use of targeted therapies as a standard treatment for HER2-positive breast cancer. In breast cancer, it is well known that HER2 overexpression is an independent prognostic factor and a predictive factor for treatment with trastuzumab. ${ }^{5,6}$ It was also showed that trastuzumab combined with chemotherapy increased the life-span of 4.2 months in HER2-positive metastatic GC patients. ${ }^{7}$ Accordingly, the relationship between the expression of HER2 and the prognosis of GC, which should be fundamental to support the value of trastuzumab as a targeted therapeutic drug for gastric cancer, has concerned many researchers. However, the results from related literature is inconsistent and requires further investigation.

Ki67, another important indicator in the molecular type of breast cancer, is also a prognostic biomarker in GC. Molecular classification provides an excellent prognostic and predictive significance in breast cancer, which were classified into four subtypes: luminal A, luminal B, HER2/neu, and basal cell-like. ${ }^{8}$ This approach uses immunohistochemical definitions of estrogen and progesterone receptors, the detection of overexpression and/or amplification of the HER2 oncogene, and Ki67 labeling index as the means of identifying tumor subtypes. ${ }^{8}$ Numerous studies reported that luminal A displayed the highest OS, followed by luminal B, HER2, and basal-like. ${ }^{9-11}$ Aberrant expression of Ki67 often depicts various malignant tumors, including breast cancer and GC. ${ }^{12,13}$ Currently, there is little research available on the molecular classification of GC. Therefore, our research objective focuses on survival analysis of the varying HER2 and Ki67 status, as well as their combinations. We divided 1663 patients with GC into four groups, based on the status of HER2 and Ki67, to explore their associations with the prognosis of GC, which may provide important information on the clinical application of trastuzumab and prognosis prediction for different GC patients.

\section{Materials And Methods}




\section{Patient and Data Collection}

The study flowchart was showed in Fig. 1. From January 2013 to December 2018, a total of 3769 consecutive cases of primary stomach or gastroesophageal junction cancer patients were recruited from the General Hospital of Ningxia Medical University of China. This study included 2062 patients after excluding 1707 patients, who exhibited residual GC and recurrence of GC combined with other tumors. Patients who received preoperative adjuvant chemotherapy or did not undergo immunohistochemistry (IHC) analysis were also excluded from the study. We retrospectively retrieved the demographic and clinicopathological features of each patient from the medical records. Demographic information, including age, gender, ethnicity, cigarette smoking and alcohol history, and blood type were collected. Clinical pathological data, including surgical method, tumor location and size, Bormann's type, tumor differentiation, Lauren classification, extent of vessel invasion, TMN stage, and adjuvant chemoradiotherapy were collected. All resection specimens were pathologically staged according to the seventh edition of the AJCC TNM classification system. ${ }^{14}$ Patient follow-up was conducted via telephone call or text message to obtain patient survival information until January 24th, 2019. The study endpoint was OS, which was regarded as the number of months from the date of admission to the date of death or last follow-up. The study was approved by the Ethical Committee of Ningxia Medical University of China (No. 2017-013).

\section{Tissue Processing And IHC Staining}

Surgically resected primary gastric tumor tissues were sent to the Department of Pathology, the General Hospital of Ningxia Medical University in China. IHC staining was carried out using HER2 (mouse anti-human monoclonal antibody, ZSGB-BIO, Beijing, China) and Ki-67(mouse anti-human monoclonal antibody, ZSGB-BIO, Beijing, China) as the primary antibody. Tissue specimens were fixed in 10\% neutral buffered formalin, embedded in paraffin, and serially sectioned ( $4 \mu \mathrm{m}$ thick). Sections were immersed in fresh xylene for 10 min to remove residual paraffin and were deparaffinized three times in $100 \%$ xylene for 10 min, and then hydrated three times in 100\%, 95\%, and 75\% alcohol for 3 min. Sections were boiled for 2 min in EDTA antigen retrieval solution $(\mathrm{pH}=9.0)$ and then naturally cooled at room temperature. Sections were immersed with $3 \%$ hydrogen peroxide for $10 \mathrm{~min}$ to eliminate endogenous peroxidase activity, and then washed three times with phosphate-buffered saline (PBS). Next, sections were incubated with HER2 and Ki-67 for $60 \mathrm{~min}$ at $37^{\circ} \mathrm{C}$. After rinsing three times with PBS, sections were incubated with sheep anti-mouse IgG polymer (ZSGB-BIO, Beijing, China) for 30 min at room temperature. After rinsing three times with PBS, sections were incubated with freshly prepared DAB coloring solution for $10 \mathrm{~min}$ at room temperature. Thereafter, sections were lightly stained with hematoxylin for 20 seconds. Finally, after dehydrating, sections were sealed with neutral gum.

\section{Immunohistochemical Assessment}

We applied the HER2 IHC scoring system for gastric cancer amended by Hoffmann et al.. ${ }^{15}$ HER2 expression was scored as follows: No membranous staining or staining in $<10 \%$ of all neoplastic cells was defined as 0 ; Faint or barely noticeable staining in $\geq 10 \%$ of neoplastic cells was defined as

$1+$; Mild to moderate basolateral or complete membranous staining in $\geq 10 \%$ of neoplastic cells was defined as $2+$; Strong or basolateral or complete membranous staining $\geq 10 \%$ of neoplastic cells was defined as $3+$. Specimens with an IHC scoring of 0 or $1+$ were classified as low HER 2 expression (abbreviated as HER2 ${ }^{\text {low }}$ ), those with IHC 2 + were classified as "HER2 equivocally", and those with IHC $3+$ were classified as high HER2 expression (abbreviated as HER2 ${ }^{\text {high }}$ ).

Ki67 expression was scored as follows: Ki67 positive tumor cells exhibited brownish-yellow granules located in their nucleus. Specimens in which the GC cells were unstained or in which $<10 \%$ of the GC cells were stained were classified as negative $(-)$ and specimens in which $\geq 10 \%$ of the GC cells were positive were classified as positive; $10-25 \%$ positive GC cells was scored $+; 26-50 \%,++; 51-75 \%$, +++ ; and $>75 \%$, ++++. Patients with tissue scores of - + , or + + were graded as low Ki67 expression (abbreviated as Ki67 low); those scored +++ or ++++ Ki67 expression were graded as high Ki67 expression (abbreviated as Ki67 ${ }^{\text {high }}$ ).

\section{Statistical analysis}

Data analysis was conducted using SPSS software (Version 17, Chicago, IL, USA). The chi-squared test was performed to analyze the relationship between the expression of HER2 and Ki67 and each variable. The Kaplan-Meier method was carried out to estimate survival rates, and survival curves were compared with the log-rank test. The hazard ratio (HR) and 95\% confidence intervals (Cls) were estimated with a Cox proportional hazard model. Multivariate Cox regression analysis was conducted to adjust for potential confounding factors. $P$ values $<0.05$ is considered statistically significant.

\section{Results}

\section{Clinical information of the study participants}

In total, 2062 patients were involved in this study, including 1626 males and 436 females, with the male-to-female ratio being $3.72: 1$. The mean age of all GC patients was 59 years old, ranging from 15 to 91 years old. According to histologic differentiation of GC, 1017 cases (52.1\%) displayed a

Page $3 / 15$ 
well or moderately differentiated type and 936 cases (47.9\%) displayed a poorly differentiated type. According to the Lauren classification, we found 229 intestinal-type cases (46.0\%), 123 diffuse-type (24.7\%), and 146 mixed-type (29.3\%). In terms of T stages, there were 339 T1 cases (16.8\%), 288 T2 cases (14.3\%), 264 T3 cases (13.0\%), and 1128 T4 cases (55.9\%). A total of 1209 (59.9\%) patients presented with lymph node metastases, and the others did not. Regarding the tumor stage, 1000 (49.5\%) patients presented with stage I and II, and 1021 (50.5\%) patients with stage III and IV (missing value 41). Additional details are summarized in Supplementary Table 1.

\section{Expression Of HER2 And Ki67 And Clinicopathological Characteristics}

HER2 and Ki67 protein expression status was determined by IHC (Fig. 2). According to the IHC results, there were 1521 HER2 ${ }^{\text {low }}$ expression cases (73.7\%), 351 HER2 equivocally expression cases (17.0\%), and 190 HER2 ${ }^{\text {high }}$ expression cases (9.2\%). There were 1289 cases (64.3\%) with Ki67 high expression and 715 cases (35.7\%) with Ki67 low expression (Fig. 1; Supplementary Table 1). According to the combination of HER2 and Ki67 expression levels, 563 cases belonged to HER2 $2^{\text {low }} / \mathrm{Ki} 67^{\mathrm{low}}$ group; 914 cases belonged to HER2 ${ }^{\text {low }} / \mathrm{Ki} 67^{\text {high }}$ group; 53 cases belonged to HER2 ${ }^{\text {high }} /$ Ki67 $^{\text {low }}$ group; and 133 cases belonged to HER2 ${ }^{\text {high }} / \mathrm{Ki} 67^{\text {high }}$ group. The four groups differ significantly when comparing sex $(P=0.020)$, tumor location $(P<0.001)$, tumor differentiation $(P<0.001)$ and Lauren type $(P<0.001)$. More details are summarized in Table 1. 
Table 1

Distribution characteristics of demographic and clinicopathological variables in patients with gastric cancer in different expression of HER2 and Ki67

\begin{tabular}{|c|c|c|c|c|c|c|}
\hline Variates & Total No.(\%) & HER2 $2^{\text {low }} / \mathrm{Ki} 67^{\text {low }}$ & HER2 ${ }^{\text {low }} / \mathrm{Ki} 67^{\text {high }}$ & HER2 ${ }^{\text {high }} /$ Ki67low & HER2 ${ }^{\text {high }} / K i 67^{\text {high }}$ & $P$ \\
\hline & & $\mathrm{n}=563$ No.(\%) & $\mathrm{n}=914 \mathrm{No} .(\%)$ & $\mathrm{n}=53$ No.(\%) & $n=133$ No.(\%) & \\
\hline \multicolumn{7}{|l|}{ Age } \\
\hline$<60$ & $841(50.6)$ & $304(54.0)$ & $452(49.5)$ & $22(41.5)$ & $63(47.4)$ & 0.146 \\
\hline$\geq 60$ & $822(49.4)$ & $259(46.0)$ & $462(50.5)$ & $31(58.5)$ & $70(52.6)$ & \\
\hline \multicolumn{7}{|l|}{ Sex } \\
\hline Male & $1304(78.4)$ & $420(74.6)$ & $733(80.2)$ & $47(88.7)$ & $104(78.2)$ & 0.020 \\
\hline Female & $359(21.6)$ & $143(25.4)$ & $181(19.8)$ & $6(11.3)$ & $29(21.8)$ & \\
\hline \multicolumn{7}{|l|}{ Ethnicity } \\
\hline Han & $1317(79.2)$ & $453(80.5)$ & 715 (78.3) & $43(81.1)$ & $106(79.7)$ & 0.772 \\
\hline Hui & $345(20.8)$ & $110(19.5)$ & $198(21.7)$ & $10(18.9)$ & $27(20.3)$ & \\
\hline \multicolumn{7}{|l|}{ Smoking } \\
\hline No & $952(57.2)$ & $331(58.8)$ & $518(56.7)$ & $28(52.8)$ & $75(56.4)$ & 0.769 \\
\hline Yes & $711(42.8)$ & $232(41.2)$ & $396(43.3)$ & $25(47.2)$ & $58(43.6)$ & \\
\hline \multicolumn{7}{|l|}{ Drinking } \\
\hline No & $1340(80.7)$ & $448(79.6)$ & $740(81.1)$ & $44(83.0)$ & $108(81.2)$ & 0.885 \\
\hline Yes & $321(19.3)$ & $115(20.6)$ & $172(18.9)$ & $9(17.0)$ & $25(18.8)$ & \\
\hline \multicolumn{7}{|l|}{ ABO } \\
\hline A & $483(29.3)$ & $164(29.3)$ & $262(29.0)$ & $19(35.8)$ & $38(29.0)$ & 0.860 \\
\hline B & $528(32.1)$ & $176(31.5)$ & $292(32.3)$ & $18(34.0)$ & $42(32.1)$ & \\
\hline$A B$ & $153(9.3)$ & $60(10.7)$ & $80(8.8)$ & $2(3.8)$ & $11(8.4)$ & \\
\hline 0 & $483(29.3)$ & $159(28.4)$ & 270 (29.9) & $14(26.4)$ & $40(30.5)$ & \\
\hline \multicolumn{7}{|l|}{ Surgical method } \\
\hline Radical & $1579(96.3)$ & $534(96.2)$ & 864 (95.9) & $52(98.1)$ & $129(98.5)$ & 0.451 \\
\hline Palliative & $61(3.7)$ & $21(3.8)$ & $37(4.1)$ & $1(1.9)$ & $2(1.5)$ & \\
\hline \multicolumn{7}{|l|}{ Bormann' type } \\
\hline type I & $252(15.7)$ & 83(15.3) & $135(15.3)$ & $11(21.6)$ & $23(17.6)$ & 0.728 \\
\hline type II & $372(23.2)$ & $137(25.3)$ & $197(22.3)$ & 9 (17.6) & $29(22.1)$ & \\
\hline type III & $557(34.7)$ & $172(31.8)$ & $320(36.2)$ & $18(35.3)$ & $47(35.9)$ & \\
\hline type IV & $425(26.5)$ & $149(27.5)$ & $231(26.2)$ & $13(25.5)$ & $32(24.4)$ & \\
\hline \multicolumn{7}{|l|}{ Tumor location } \\
\hline Upper & $394(26.7)$ & $114(22.4)$ & $220(27.5)$ & $19(42.2)$ & $41(34.5)$ & $<0.001$ \\
\hline Middle & $362(24.6)$ & $150(29.5)$ & $169(21.1)$ & $9(20.0)$ & $34(28.6)$ & \\
\hline Lower & $717(48.7)$ & $245(48.1)$ & $411(51.4)$ & $17(37.8)$ & $44(37.0)$ & \\
\hline \multicolumn{7}{|l|}{ Tumor size } \\
\hline$<5$ & $902(56.3)$ & $316(58.6)$ & $494(55.9)$ & $24(45.3)$ & $68(53.1)$ & 0.224 \\
\hline$\geq 5$ & 701 (43.7) & $223(41.4)$ & $389(44.1)$ & $29(54.7)$ & $60(46.9)$ & \\
\hline \multicolumn{7}{|l|}{ Pathology type } \\
\hline Adenocarcinoma & 1164 (91.3) & $385(91.0)$ & 648 (91.7) & $36(90.0)$ & $95(90.5)$ & 0.956 \\
\hline
\end{tabular}




\begin{tabular}{|c|c|c|c|c|c|c|}
\hline Variates & Total No.(\%) & HER2 $2^{\text {low }} /$ Ki67 low & HER2 ${ }^{\text {low }} /$ Ki $7^{\text {high }}$ & HER2 $2^{\text {high }} /$ Ki67low & HER2 ${ }^{\text {high }} /$ Ki67 $7^{\text {high }}$ & $P$ \\
\hline signet-ring cell & $60(4.7)$ & $19(4.5)$ & $32(4.5)$ & $3(7.5)$ & $6(5.7)$ & \\
\hline mucinous & $51(4.0)$ & $19(4.5)$ & $27(3.8)$ & $1(2.5)$ & $4(3.8)$ & \\
\hline \multicolumn{7}{|c|}{ Tumor differentiation } \\
\hline Well & $169(10.7)$ & $50(9.3)$ & $79(9.2)$ & $16(30.8)$ & $24(18.2)$ & $<0.001$ \\
\hline Moderate & $625(39.7)$ & $191(35.7)$ & $338(39.5)$ & $23(44.2)$ & $73(55.3)$ & \\
\hline Poor & $781(49.6)$ & $294(55.0)$ & $439(51.3)$ & $13(25.0)$ & 35 (26.5) & \\
\hline \multicolumn{7}{|l|}{ Lauren type } \\
\hline Intestinal type & $188(44.2)$ & $58(36.3)$ & $89(41.2)$ & 17 (89.5) & $24(80.0)$ & $<0.001$ \\
\hline Diffuse type & $113(26.6)$ & $50(31.3)$ & $60(27.8)$ & $1(5.3)$ & $2(6.7)$ & \\
\hline Mixed type & $124(29.2)$ & $52(32.5)$ & $67(31.0)$ & $1(5.3)$ & $4(13.3)$ & \\
\hline \multicolumn{7}{|l|}{ Vessel invasive } \\
\hline No & $330(56.3)$ & $109(54.8)$ & $186(56.7)$ & $14(77.8)$ & $21(51.2)$ & 0.260 \\
\hline Yes & $256(43.7)$ & $90(45.2)$ & $142(43.3)$ & $4(22.2)$ & $20(48.8)$ & \\
\hline \multicolumn{7}{|l|}{ T stage } \\
\hline $\mathrm{T} 1$ & $270(16.5)$ & $99(17.8)$ & $145(16.1)$ & $8(15.1)$ & $18(13.8)$ & 0.163 \\
\hline T2 & $241(14.7)$ & 65 (11.7) & $142(15.8)$ & $7(13.2)$ & $27(20.8)$ & \\
\hline T3 & $213(13.0)$ & $69(12.4)$ & $114(12.7)$ & $11(20.8)$ & $19(14.6)$ & \\
\hline $\mathrm{T} 4$ & $912(55.7)$ & $322(58.0)$ & $497(55.3)$ & $27(50.9)$ & $66(50.8)$ & \\
\hline \multicolumn{7}{|l|}{$\mathrm{N}$ stage } \\
\hline No & $648(39.8)$ & $236(42.8)$ & $342(38.1)$ & $25(47.2)$ & $45(34.9)$ & 0.129 \\
\hline Yes & $982(60.2)$ & $315(57.2)$ & $555(61.9)$ & $28(52.8)$ & $84(65.1)$ & \\
\hline \multicolumn{7}{|l|}{ M stage } \\
\hline No & 1565 (95.7) & $530(95.3)$ & $857(95.5)$ & $51(96.2)$ & $127(97.7)$ & 0.678 \\
\hline Yes & $71(4.3)$ & $26(4.7)$ & $40(4.5)$ & $2(3.8)$ & $3(2.3)$ & \\
\hline \multicolumn{7}{|l|}{ TNM stage } \\
\hline$I+\|$ & $805(49.2)$ & $279(50.2)$ & $428(47.7)$ & $28(52.8)$ & $70(53.8)$ & 0.492 \\
\hline III + IV & $831(50.8)$ & $277(49.8)$ & $469(52.3)$ & $25(47.2)$ & $60(46.2)$ & \\
\hline \multicolumn{7}{|c|}{ Adjuvant chemotherapy } \\
\hline No & 1019 (61.3) & $352(62.5)$ & $548(60.0)$ & $34(64.2)$ & 85 (63.9) & 0.656 \\
\hline Yes & $644(38.7)$ & $211(37.5)$ & $366(40.0)$ & 19(35.8) & $48(36.1)$ & \\
\hline \multicolumn{7}{|c|}{ Adjuvant radiotherapy } \\
\hline No & $1629(98.0)$ & $549(97.5)$ & $895(97.9)$ & $52(98.1)$ & $133(100)$ & 0.342 \\
\hline Yes & $34(2.0)$ & $14(2.5)$ & $19(2.1)$ & $1(1.9)$ & 0 & \\
\hline
\end{tabular}

\section{Relationship Between HER2 And Ki67 Expression Level And OS}

In this retrospective study, a total of 1827 patients were successfully followed for survival analysis. The median follow-up time was 34.1 months (range: $0.2-74.6$ months), and 575 patients (27.9\%) died of cancer, while the remaining 1252 patients (68.5\%) were alive at the end of the follow-up time. The OS between 1521 HER2 ${ }^{\text {low }}$ expression patients and 190 HER2 ${ }^{\text {high }}$ expression patients was compared and no statistically significant survival difference was found for OS rates (60.6\% vs 61.5\%, $P=0.748)$ (Supplementary Fig. 1a). The Ki67 high expression was associated with significantly poorer survival, with the statistical $P$ values around the cut-off value for OS $(62.9 \%$ vs $59.6 \%, P=0.063)$. Based on the combined 
expression of HER2 and Ki67, no significant survival difference was found among the four groups $(63.2 \%, 59.7 \%, 69.8 \%$, and $57.8 \%, P=0.281)$ (Fig. 3a-3b). The 1-, 3-, and 5-year OS rates of different patient groups are showed in Supplementary Table 2.

\section{Univariate And Multivariate Analyses Of OS}

In the univariate analysis, we found that patients over 60 years old had reduced OS rate compared with younger patients in HER2 ${ }^{\text {low }} / \mathrm{Ki} 67^{\text {low }}$ group (63.8\% vs $62.2 \%, P=0.035)$ (Supplementary Fig. 1 b), HER2 ${ }^{\text {low }} /$ Ki67 $7^{\text {high }}$ group $(62.6 \%$ vs $56.5 \%, P<0.001)$ (Supplementary Fig. $\left.1 \mathrm{c}\right)$, and HER2 ${ }^{\text {high }} / \mathrm{Ki} 7^{\text {high }}$ group $(69.8 \%$ vs $45.7 \%, P<0.001)$ (Supplementary Fig. 1 d). We observed that patients with a smoking history significantly reduced the OS rate of HER2 ${ }^{\text {low }} /$ Ki67 $^{\text {low }}$ group $(66.2 \%$ vs $58.6 \%, P=0.080)$ (Supplementary Fig. 1 e) and HER2 $2^{\text {high }} /$ Ki67 $^{\text {low }}$ group $(78.6 \%$ vs $60.0 \%, P=0.652)$ (Supplementary Fig. 1f). The OS differences which reach statistical levels between HER2 ${ }^{\text {low }} /$ Ki $67^{\text {low }}$ and HER2 ${ }^{\text {low }} /$ Ki67 $^{\text {high }}$ groups for non-smokers, blood type A, AB, and 0 , adenocarcinoma, metastasis and chemotherapy were also observed in Fig. 3c-3i $(P<0.05)$. Additional details are provided in Table 2 
Table 2

Univariate survival analysis comparing the impact of demographic and clinicopathological characteristic among four groups based on HER2 and Ki67 expression

\begin{tabular}{|c|c|c|c|c|c|c|c|c|c|}
\hline Variates & HER2 ${ }^{\text {low }} /$ Ki67low & HER2 $2^{\text {low }} /$ Ki $7^{\text {high }}$ & HER2 ${ }^{\text {high }} /$ Ki $67^{\text {low }}$ & HER2 ${ }^{\text {high }} / K i 67^{\text {high }}$ & $P^{a}$ & $P^{b}$ & $P^{c}$ & $P^{d}$ & $p^{e}$ \\
\hline Age & & & & & 0.160 & 0.179 & 0.468 & 0.179 & 0.312 \\
\hline$<60$ & 63.8 & 62.6 & 68.2 & 69.8 & 0.637 & 0.602 & 0.645 & 0.602 & 0.91 \\
\hline$\geq 60$ & 62.2 & 56.5 & 71.0 & 45.7 & 0.148 & 0.044 & 0.213 & 0.044 & 0.123 \\
\hline Sex & & & & & 0.091 & 0.265 & 0.547 & 0.265 & 0.253 \\
\hline Male & 64.8 & 61.0 & 66.0 & 60.9 & 0.115 & 0.702 & 0.965 & 0.702 & 0.439 \\
\hline Female & 58.0 & 53.6 & 100.0 & 44.8 & 0.518 & 0.068 & 0.146 & 0.068 & 0.266 \\
\hline Ethnicity & & & & & 0.119 & 0.252 & 0.530 & 0.252 & 0.299 \\
\hline Han & 63.8 & 60.4 & 69.8 & 60.4 & 0.137 & 0.577 & 0.762 & 0.577 & 0.446 \\
\hline Hui & 60.0 & 56.6 & 70.0 & 44.4 & 0.595 & 0.190 & 0.451 & 0.19 & 0.531 \\
\hline Smoking & & & & & 0.115 & 0.242 & 0.466 & 0.242 & 0.275 \\
\hline No & 66.2 & 57.3 & 78.6 & 54.7 & 0.011 & 0.167 & 0.661 & 0.167 & 0.036 \\
\hline Yes & 58.6 & 62.4 & 60.0 & 60.3 & 0.597 & 0.878 & 0.555 & 0.878 & 0.886 \\
\hline Drinking & & & & & 0.115 & 0.230 & 0.540 & 0.23 & 0.289 \\
\hline No & 62.3 & 58.5 & 72.7 & 53.7 & 0.147 & 0.088 & 0.354 & 0.088 & 0.163 \\
\hline Yes & 66.1 & 64.0 & 55.6 & 72.0 & 0.531 & 0.213 & 0.548 & 0.213 & 0.455 \\
\hline $\mathrm{ABO}$ & & & & & 0.175 & 0.284 & 0.410 & 0.284 & 0.391 \\
\hline A & 55.5 & 64.1 & 68.4 & 52.6 & 0.018 & 0.803 & 0.391 & 0.803 & 0.123 \\
\hline B & 61.9 & 63.0 & 66.7 & 54.8 & 0.704 & 0.322 & 0.836 & 0.322 & 0.689 \\
\hline$A B$ & 65.0 & 57.5 & 0.0 & 45.5 & 0.030 & 0.164 & 0.004 & 0.164 & 0.008 \\
\hline 0 & 70.4 & 53.7 & 85.7 & 67.5 & 0.001 & 0.153 & 0.209 & 0.153 & 0.002 \\
\hline Surgical method & & & & & 0.121 & 0.279 & 0.658 & 0.279 & 0.308 \\
\hline Radical & 65.2 & 61.7 & 71.2 & 58.1 & 0.099 & 0.211 & 0.599 & 0.211 & 0.945 \\
\hline Palliative & 23.8 & 21.6 & 0.0 & 0.0 & 0.938 & 0.317 & 0.780 & 0.317 & - \\
\hline Bormann' type & & & & & 0.145 & 0.280 & 0.456 & 0.280 & 0.345 \\
\hline type I & 66.3 & 68.9 & 90.9 & 69.6 & 0.483 & 0.210 & 0.244 & 0.210 & 0.562 \\
\hline type II & 70.8 & 65.0 & 66.7 & 65.5 & 0.242 & 0.815 & 0.915 & 0.815 & 0.604 \\
\hline type III & 61.0 & 56.3 & 66.7 & 46.8 & 0.348 & 0.538 & 0.985 & 0.538 & 0.658 \\
\hline type IV & 55.7 & 53.2 & 61.5 & 56.3 & 0.301 & 0.627 & 0.605 & 0.627 & 0.644 \\
\hline Tumor location & & & & & 0.543 & 0.236 & 0.560 & 0.236 & 0.566 \\
\hline Upper & 62.3 & 50.5 & 73.7 & 46.3 & 0.032 & 0.346 & 0.737 & 0.346 & 0.122 \\
\hline Middle & 60.0 & 67.5 & 77.8 & 64.7 & 0.190 & 0.364 & 0.283 & 0.346 & 0.453 \\
\hline Lower & 66.9 & 65.5 & 64.7 & 59.1 & 0.984 & 0.795 & 0.765 & 0.795 & 0.801 \\
\hline Tumor size & & & & & 0.426 & 0.204 & 0.283 & 0.204 & 0.514 \\
\hline$<5$ & 73.1 & 68.4 & 79.2 & 63.2 & 0.184 & 0.402 & 0.896 & 0.402 & 0.445 \\
\hline$\geq 5$ & 50.2 & 50.6 & 62.1 & 50.0 & 0.953 & 0.338 & 0.237 & 0.338 & 0.677 \\
\hline Pathology type & & & & & 0.029 & 0.115 & 0.270 & 0.115 & 0.06 \\
\hline Adenocarcinoma & 63.6 & 58.0 & 77.8 & 57.9 & 0.021 & 0.177 & 0.443 & 0.177 & 0.067 \\
\hline signet-ring cell & 63.2 & 53.1 & 66.7 & 83.3 & 0.971 & 0.299 & 0.337 & - & 0.355 \\
\hline
\end{tabular}




\begin{tabular}{|c|c|c|c|c|c|c|c|c|c|}
\hline Variates & HER2 $2^{\text {low }} /$ Ki67low & HER2 ${ }^{\text {low }} /$ Ki $67^{\text {high }}$ & HER2 ${ }^{\text {high }} /$ Ki67low & HER2 $2^{\text {high }} /$ Ki67 $7^{\text {high }}$ & $P^{a}$ & $P^{b}$ & $P^{c}$ & $P^{d}$ & $p^{e}$ \\
\hline mucinous & 52.6 & 59.3 & 100.0 & 25.0 & 0.969 & - & 0.532 & 0.299 & 0.259 \\
\hline $\begin{array}{l}\text { Tumor } \\
\text { differentiation }\end{array}$ & & & & & 0.137 & 0.406 & 0.934 & 0.406 & 0.393 \\
\hline Well & 76.0 & 58.2 & 75.0 & 79.2 & 0.022 & 0.885 & 0.482 & 0.885 & 0.088 \\
\hline Moderate & 66.5 & 62.1 & 73.9 & 47.9 & 0.847 & 0.143 & 0.667 & 0.143 & 0.194 \\
\hline Poor & 57.5 & 57.2 & 53.8 & 62.9 & 0.313 & 0.613 & 0.839 & 0.613 & 0.64 \\
\hline Lauren type & & & & & 0.240 & 0.142 & 0.345 & 0.142 & 0.394 \\
\hline Intestinal type & 69.0 & 59.6 & 70.6 & 75.0 & 0.577 & 0.581 & 0.915 & 0.581 & 0.656 \\
\hline Diffuse type & 54.0 & 61.7 & 0.0 & 50.0 & 0.936 & 0.157 & 0.003 & 0.157 & 0.137 \\
\hline Mixed type & 73.1 & 56.7 & 0.0 & 75.0 & 0.160 & 0.046 & 0.001 & 0.046 & 0 \\
\hline Vessel invasive & & & & & 0.612 & 0.597 & 0.297 & 0.597 & 0.704 \\
\hline No & 60.6 & 67.2 & 71.4 & 71.4 & 0.435 & 0.909 & 0.667 & 0.909 & 0.652 \\
\hline Yes & 56.7 & 59.9 & 100.0 & 65.0 & 0.949 & 0.268 & 0.210 & 0.268 & 0.825 \\
\hline T stage & & & & & 0.122 & 0.309 & 0.591 & 0.309 & 0.3 \\
\hline $\mathrm{T} 1$ & 86.9 & 84.8 & 75.0 & 77.8 & 0.568 & 0.232 & 0.022 & 0.232 & 0.17 \\
\hline $\mathrm{T} 2$ & 76.9 & 75.4 & 85.7 & 74.1 & 0.884 & 0.429 & 0.295 & 0.429 & 0.686 \\
\hline T3 & 58.0 & 57.0 & 81.8 & 63.2 & 0.909 & 0.373 & 0.175 & 0.373 & 0.514 \\
\hline T4 & 54.3 & 49.1 & 59.3 & 42.4 & 0.102 & 0.326 & 0.938 & 0.326 & 0.258 \\
\hline $\mathrm{N}$ stage & & & & & 0.210 & 0.522 & 0.862 & 0.522 & 0.604 \\
\hline No & 78.0 & 77.5 & 84.0 & 68.9 & 0.970 & 0.819 & 0.745 & 0.819 & 0.836 \\
\hline Yes & 53.3 & 49.4 & 57.1 & 50.0 & 0.162 & 0.542 & 0.690 & 0.542 & ' 0.470 \\
\hline M stage & & & & & 0.116 & 0.278 & 0.572 & 0.278 & 0.302 \\
\hline No & 64.0 & 61.8 & 72.5 & 57.5 & 0.335 & 0.216 & 0.469 & 0.216 & '0.500 \\
\hline Yes & 50.0 & 17.5 & 0.0 & 33.3 & 0.032 & 0.515 & 0.444 & 0.515 & 0.18 \\
\hline TNM stage & & & & & 0.178 & 0.380 & 0.837 & 0.380 & 0.431 \\
\hline$I+I I$ & 77.1 & 75.2 & 85.7 & 72.9 & 0.563 & 0.978 & 0.973 & 0.978 & 0.934 \\
\hline III + IV & 49.5 & 45.8 & 52.0 & 38.3 & 0.223 & 0.302 & 0.821 & 0.302 & 0.402 \\
\hline $\begin{array}{l}\text { Adjuvant } \\
\text { chemotherapy }\end{array}$ & & & & & 0.107 & 0.237 & 0.568 & 0.237 & 0.283 \\
\hline No & 65.9 & 59.7 & 73.5 & 54.1 & 0.039 & 0.221 & 0.682 & 0.221 & 0.127 \\
\hline Yes & 58.3 & 59.3 & 63.2 & 62.5 & 0.972 & 0.767 & 0.684 & 0.767 & 0.975 \\
\hline $\begin{array}{l}\text { Adjuvant } \\
\text { radiotherapy }\end{array}$ & & & & & 0.081 & & 0.524 & & \\
\hline No & 63.0 & 60.3 & 69.2 & 57.1 & 0.216 & 0.288 & 0.595 & 0.288 & 0.455 \\
\hline Yes & 64.3 & 21.1 & 100.0 & 0.0 & 0.018 & - & 0.502 & - & 0.033 \\
\hline \multicolumn{10}{|c|}{$\mathrm{P}^{a}$ value is calculated using the log-rank test between $\mathrm{HER} 2^{\mathrm{low}} / \mathrm{Ki} 67^{\text {low }}$ and HER2 $2^{\text {low }} / \mathrm{Ki} 67^{\text {high }}$ for different variables. } \\
\hline \multicolumn{10}{|c|}{$\mathrm{P}^{b}$ value is calculated using the log-rank test between HER2 ${ }^{\text {high }} / \mathrm{Ki} 67^{\text {low }}$ and HER $2^{\text {high }} / \mathrm{Ki} 67^{\text {high }}$ for different variables. } \\
\hline \multicolumn{10}{|c|}{$\mathrm{P}^{c}$ value is calculated using the log-rank test between HER2 $2^{\mathrm{low}} / \mathrm{Ki} 67^{\mathrm{low}}$ and $\mathrm{HER} 2^{\text {high }} / \mathrm{Ki} 67^{\mathrm{low}}$ for different variables. } \\
\hline \multicolumn{10}{|c|}{$\mathrm{P}^{d}$ value is calculated using the log-rank test between HER2 $2^{\text {high }} / \mathrm{Ki} 67^{\text {low }}$ and HER2 $2^{\text {high }} / \mathrm{Ki} 67^{\text {high }}$ for different variables. } \\
\hline
\end{tabular}


According to multivariate Cox regression analysis, Ki67 (P=0.048), sex $(P=0.015)$, T stage $(P<0.001)$, N stage $(P<0.001)$, TNM stage $(P=0.012)$, and adjuvant chemotherapy $(P<0.001)$ were regarded as significant and independent risk factors for overall survival in GC. Additional details are summarized in Table 3.

Table 3

Multivariate survival analysis of independent prognostic factors for patients with

\begin{tabular}{|llll|}
\hline \multicolumn{4}{c}{ gastric cancer } \\
\hline Variables & Category & HR $(95 \% \mathrm{Cl})$ & $P$ \\
\hline total & & & \\
\hline HER7 & High expression & $1.225(1.002-1.498)$ & 0.048 \\
\hline Sex & Positive & $0.991(0.737-1.333)$ & 0.953 \\
\hline T stage & Male & $1.326(1.056-1.667)$ & 0.015 \\
\hline N stage & T3-4 & $2.774(1.897-4.056)$ & $<0.001$ \\
\hline TNM stage & N1-4 & $2.009(1.402-2.879)$ & $<0.001$ \\
\hline Adjuvant chemotherapy & Yes & $1.566(1.104-2.222)$ & 0.012 \\
\hline
\end{tabular}

\section{Discussion}

The treatment of advanced GC is still a challenge despite various clinical therapies. Molecular target therapy, which has been proved to be very effective in breast cancer, might present a potential therapeutic option for GC as well. In this study, we analyzed 2062 GC patients with resectable GC to identify the relationship between the expression of HER2 and Ki67 and the resultant prognosis.

Firstly, the rate of high HER2 expression in our study was found to be 9.2\% (190 of 2062) using IHC, which was consistent with most previous reports (4.4-53.4\%). ${ }^{16}$ Moreover, the OS rates was compared between 1521 low HER2 expression patients and 190 high HER2 expression patients and the results did not indicate a significant survival difference for 1 -year OS (60.6\% vs $61.5 \%)$, 3-year OS (51.3\% vs $53.8 \%$ ) or 5 -year OS (26.6\% vs $27.6 \%$ ), which was consistent with the analysis in 810 Korean GC patients. ${ }^{17}$ However, two other studies demonstrated that HER2 overexpression is associated with poor survival outcomes in 100 Iranian patients and 1148 Japanese cases with GC. ${ }^{18,19}$ The reasons for the inconsistent results could be partly attributed to the heterogeneity of HER2 expression among different ethnic populations or the effects of sample size. Therefore, it reminds us that cautions should be taken when using the targeted therapeutic drugs, trastuzumab for GC patients with positive HER2 expression in different populations.

Secondly, we found that the OS rate of high Ki67 expression in GC patients was lower than that the low expression group, with statistical $P$ values being around the cut-off value for 1 -year OS (63.0\% vs $59.9 \%, P=0.090)$ and 3 -year OS $(54.3 \%$ vs $51.0 \%$, $P=0.074)$, which was consistent with many other previous studies. ${ }^{20,21}$ Our cox model further supports that Ki67 acts as an independent prognostic factor in the resectable GC patients in this study.

We then divided all patients into four groups based on the expression status of HER2 and Ki67 to further compare their OS rates among four groups. Although there were no statistical differences among the OS rates of four groups, it was found that GC patients in HER2 ${ }^{\text {high }} /$ Ki67 $^{\text {low }}$ group showed the highest OS of $69.8 \%$, followed by those patients in other three groups with OS rates being $63.2 \%, 59.7 \%$, and $57.8 \%$. We then conducted subgroup analyses, which stratified the related variables. It is interesting to find that patients over 60 years old had reduced survival time compared with younger patients among three groups. It was also observed that patients with a smoking history exhibited poorer survival in HER2 ${ }^{\text {low }} / \mathrm{Ki} \mathrm{7}^{\mathrm{low}}$ and HER2 ${ }^{\text {high }} /$ Ki67 $^{\text {low }}$ groups, compared with patients without a smoking history. Moreover, it was clearly showed that the OS time in HER2 low/ Ki67 high group was significantly shorter than that in HER2 $2^{\text {low }} / K_{16} 7^{\text {low }}$ for patients with no-smoking history, blood type AB, blood type O, adenocarcinoma, metastasis, and no-adjuvant chemotherapy. However, the OS time in HER2 $2^{\text {high }} / \mathrm{Ki} 67^{\text {low }}$ group was longer than that in HER2 ${ }^{\text {low }} / \mathrm{Ki} 67^{\text {low }}$ for patients with blood type A. All these findings mentioned above support that routine evaluation of combining HER2 and Ki67 expression levels could be useful to assess patient prognosis with resectable GC.

However, our study had several limitations. Firstly, the expression level of HER2 was tested only using IHC. The cases which scored $2+$ using IHC were not confirmed using a FISH test. Accordingly, the number of HER2 positive cases were affected, which may be prone to bias. Secondly, most patients received several uncontrolled adjuvants and palliative chemotherapies, which may lead to a protective effect for patients with GC. Lastly, our data were retrieved only from a single agency, indicating that caution should be exercised when interpreting the results.

To the best of our knowledge, this is the first study to evaluate HER2 status combined with Ki67 status in 2062 patients with GC. In conclusion, this large-scale study demonstrated that low Ki67 expression is an independent prognostic factor in GC patients and various combinations of HER2 and 
Ki67 expression can affect patient prognosis.

\section{Declarations}

Ethics approval and consent to participate

The study was approved by the Ethical Committee of Ningxia Medical University prior to study conduct (No. 2017-013). Informed consent was obtained from all patients included in the study.

Consent for publication

Written informed consent for publication was obtained from all participants.

Availability of data and materials

The datasets used and/or analyzed during the current study are available from the corresponding author on reasonable request.

Competing interests

The authors declare that they have no competing interests

Funding

Wenjun Yang was supported by grants from National Natural Science Foundation of China (No. 81760525, 81160249). Yanjie You was supported by grants from National Natural Science Foundation of China (No.81860426) and the Natural Science Foundation of Ningxia, China (No.

2018AAC02016).

Authors' contributions

All authors contributed to the study conception and design. WW, JC, PC, YY, DZ, XZ, Qian Z and JW collected patient information, cancer tissues and followed up all patient survival status. YY, ZC, QZ, AM, JW, and GW did all HIC experiments. WW, ZC, QZ and GW interpreted the collected data and generated tables and figures for the data. WW and WY edited the manuscript. WY and JR supervised the study. All authors read and approved the final manuscript.

Acknowledgements

We thank Wiley for the linguistic assistance during the preparation of this manuscript. We thank all of the participants of this study.

\section{References}

1. Freddie Bray J, Ferlay I, Soerjomataram RL, Siegel LA, Torre. Ahmedin Jemal. Global Cancer Statistics 2018: GLOBOCAN Estimates of Incidence and Mortality Worldwide for 36 Cancers in 185 Countries. CA. 2018;68:394-424.

2. Chen W, Sun K, Zheng R, Zeng H, Zhang S, Xia C, et al. Cancer incidence and mortality in China, 2014. Chinese J Cancer Res. 2018;30:1-12.

3. Lin CAI, Yang B, Parkin DM, Zuofeng ZHANG. Analysis of Malignant Tumor Epidemic Trends in Several Asia-Pacific Regions. J Oncol. 2004;Issue 5:422-6.

4. Hudis CA. Drug Therapy: Trastuzumab-Mechanism of Action and Use in Clinical Practice. New Engl J Med. 2007;357:39-51.

5. Seshadri R, Firgaira FA, Horsfall DJ, McCaul K, Setlur V, Kitchen P. Clinical significance of HER-2/neu oncogene amplification in primary breast cancer. The South Australian Breast Cancer Study Group. Clin Oncol. 1993;11:1936-78.

6. Dawood S, Broglio K, Buzdar AU, Hortobagyi GN, Giordano SH. Prognosis of women with metastatic breast cancer by HER2 status and trastuzumab treatment: an institutional-based review. Clin Oncol. 2010;28:92-8.

7. Lin Shen Jian-ming, Xu Feng-yi, Feng Shun-chang, Jiao Li-wei, Wang J, Li, et al. Trastuzumab in combination with chemotherapy versus chemotherapy alone for treatment of HER2-positive advanced gastric or gastro-oesophageal junction cancer (ToGA): a phase3, open-label, randomised controlled trial. Lancet. 2010;376:687-97.

8. Goldhirsch A, Winer EP, Coates AS, Gelber RD, Piccart-Gebhart M, Thürlimann B, et al. Personalizing the treatment of women with early breast cancer: highlights of the St Gallen International expert consensus on the primary therapy of early breast cancer 2013. Ann Oncol. 2013;24:220623.

9. Antonio G, Fernández C, Chabrera M, García Font M, Fraile S, Gónzalez I, Barco, et al. Differential survival and recurrence patterns of patients operated for breast cancer according to the new immunohistochemical classification: analytical survey from 1997 to 2012 . Tumour Biol. 2013;34:2349-55.

Page $11 / 15$ 
10. Carlos S, Vallejos HL, Gómez, Wilder R, Cruz JA, Pinto RR, Dyer, Raúl Velarde, et al. Breast Cancer Classification According to Immunohistochemistry Markers: Subtypes and Association With Clinicopathologic Variables in a Peruvian Hospital Database. Clin Breast Cancer. 2010;10:294-300.

11. Elisabeth Christine Inwald M, Koller M, Klinkhammer-Schalke F, Zeman F, Hofstdter M, Gerstenhauer, et al. 4-IHC classification of breast cancer subtypes in a large cohort of a clinical cancer registry: use in clinical routine for therapeutic decisions and its effect on survival. Breast Cancer Res Tr. 2015;153:647-58.

12. Masami Morimoto Y, Bando M, Nakagawa H, Takechi T, Yoshida, et al. Immunocytochemical results for HER2 and Ki67 in breast cancer touchsmear cell specimens are reliable. Breast Cancer. 2016;23:577-82.

13. Wu A, Jia Y, Dong B, Tang L, Liu Y, Du H, et al. Apoptosis and KI 67 index correlate with preoperative chemotherapy efficacy and better predict the survival of gastric cancer patients with combined therapy. Cancer Chemoth Pharm. 2014;73:885-93.

14. Edge SB, Byrd DR, Compton CC, Fritz AG, Greene FL, Trotti A. AJCC cancer staging manual. 7th ed. New York: Springer; 2010. pp. $117-26$.

15. Hofmann M, Stoss O, Shi D, Bu“ttner R, van de Vijver M, Kim W, et al. Assessment of a HER2 scoring system for gastric cancer: result from a validation study. Histopathology. 2008;52:797-805.

16. Jørgensen JT, Hersom M. HER2 as a Prognostic Marker in gastric cancer: a systematic analysis of data from the literature. Cancer. 2012;3:13744.

17. Kim H, Seo S, Kim K, Park YH, An M, Baik H, et al. Prognostic significance of Human epidermal growth factor receptor-2 expression in patients with resectable gastric adenocarcinoma. World J Surg Oncol. 2019;17:122.

18. Aznab M, Maleksabet D, Khazaei S, Khazaei M, Rezaei M. The Role of Human Epidermal Growth Factor Receptor (HER2/neu) in the Prognosis of Patients with Gastric Cancer. Asian Pac J Cancer P. 2019;20:1989-94.

19. Yukinori Kurokawa N, Matsuura Y, Kimura S, Adachi J, Fujita H, Imamura, et al. Multicenter large-scale study of prognostic impact of HER2 expression in patients with resectable gastric cancer. Gastric Cancer. 2016;18:691-7.

20. Tzanakis NE, Peros G, Karakitsos P, Giannopoulos GA, Efstathiou SP, Rallis G, et al. Prognostic significance of p53 and Ki67 proteins expression in greek gastric cancer patients. Can J Psychiat. 2009;109:606-11.

21. Huang GL, Chen SQ, Wang DY, Wang RH, Lin LY, Chen SM, et al. High Ki67 Expression has Prognostic Value in Surgically-Resected T3 Gastric Adenocarcinoma. Clin Lab. 2016;62:141-53.

\section{Figures}




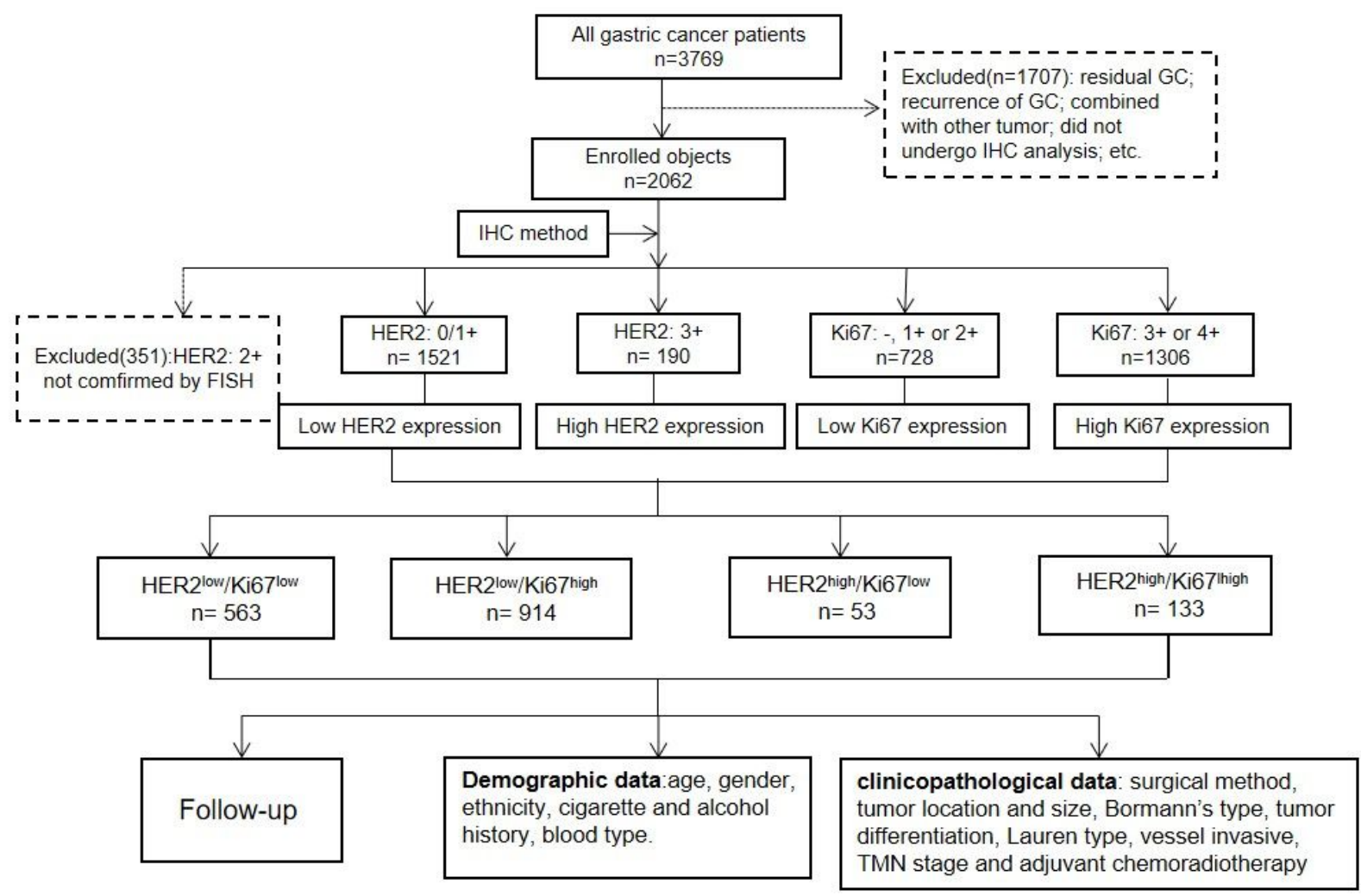

\section{Figure 1}

Study flowchart 


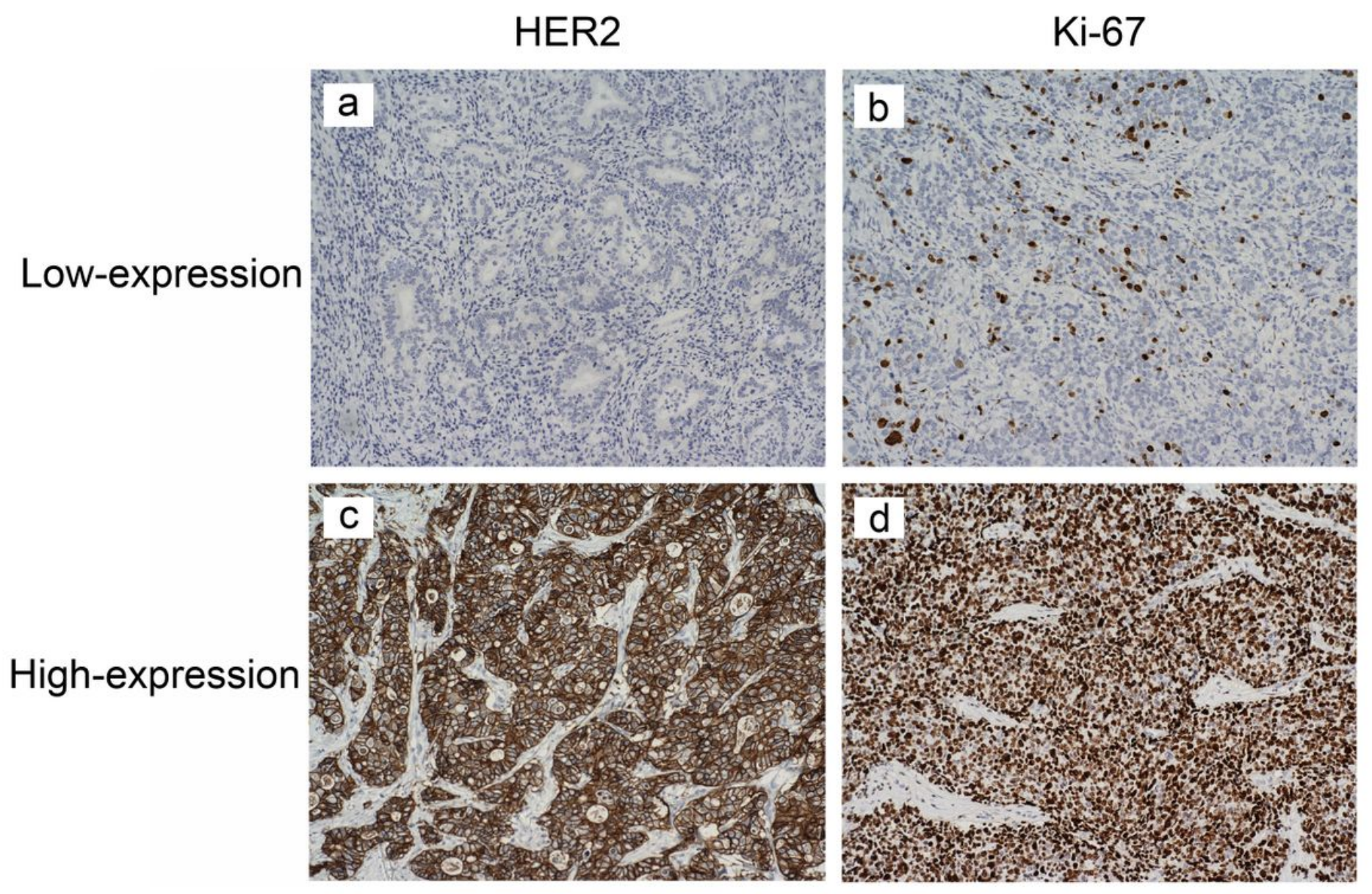

Figure 2

The expression of HER2 and Ki67 protein detected by IHC. Representative specimen with low HER2 expression (a), high HER2 expression (c) (x200), low Ki67 expression (b), and high Ki67 expression (d) (x200). 



Figure 3

Overall survival according to: a. Ki67 in 2,034 gastric cancer patients b. HER2 and Ki67 status in 1663 patients. Overall survival between HER2low/Ki67high and HER2low/Ki67low for c. Non-smokers, d, e, f. Blood type. g. Adenocarcinoma, h. Metastasis, i. No chemotherapy.

\section{Supplementary Files}

This is a list of supplementary files associated with this preprint. Click to download.

- Supplementarydata.pdf 